\title{
Evolution of the spectrum of the Hubbard model with filling
}

\author{
A.Sherman \\ Institute of Physics, University of Tartu, Riia 142, 51014 Tartu, Estonia \\ Received February 27, 2006, in final form May 12, 2006
}

The diagram technique for the one-band Hubbard model is formulated for the case of moderate to strong Hubbard repulsion. The expansion in powers of the hopping constant is expressed in terms of site cumulants of electron creation and annihilation operators. For Green's function an equation of the Larkin type is derived and solved in a one-loop approximation for the case of two dimensions and nearest-neighbor hopping. With decreasing the electron concentration in addition to the four bands observed at half-filling, a narrow band arises near the Fermi level. The dispersion of the new band, its bandwidth and the variation with filling are close to those of the spin-polaron band in the $t-J$ model.

Key words: Hubbard model, diagram technique, energy spectrum

PACS: $71.10 . F d, 71.10 .-w$

\section{Introduction}

For the last two decades the discovery of high- $T_{\mathrm{c}}$ superconductors, heavy-fermion compounds and organic conductors has revived interest in strongly correlated electron systems. One of the simplest and still realistic models in this field is the one-band Hubbard model [1-3] the twodimensional version of which has been extensively studied in connection with the cuprate perovskite superconductors. The Hamiltonian of the model reads

$$
H=\sum_{\mathbf{n} \mathbf{m} \sigma} t_{\mathbf{n m}} a_{\mathbf{n} \sigma}^{\dagger} a_{\mathbf{m} \sigma}+\frac{U}{2} \sum_{\mathbf{n} \sigma} n_{\mathbf{n} \sigma} n_{\mathbf{n},-\sigma},
$$

where $t_{\mathbf{n m}}$ is the hopping constants, the operator $a_{\mathbf{n} \sigma}^{\dagger}$ creates an electron on the $\mathbf{n}$ site of a plane square lattice with the spin projection $\sigma= \pm 1, U$ is the on-site Coulomb repulsion and the electron number operator $n_{\mathbf{n} \sigma}=a_{\mathbf{n} \sigma}^{\dagger} a_{\mathbf{n} \sigma}$. If the Coulomb repulsion dominates it is reasonable to treat this interaction exactly and the kinetic energy with the use of a perturbation expansion. Apparently the first expansion of this kind was considered in reference [4]. The further development of this approach was given in references [5-9] where the diagram technique for Hubbard operators was developed and used in investigating the Mott transition, the magnetic phase diagram and the superconducting transition in the Hubbard model.

Another, more compact version of the diagram technique was proposed in references [10-13]. In this approach the power expansion for the electron Green's function is expressed in terms of cumulants of electron operators $a_{\mathbf{n} \sigma}$ and $a_{\mathbf{n} \sigma}^{\dagger}$. Based on this diagram technique the equations of the Larkin type [15] for Green's function were derived [10,12,13]. However, the application of this approach runs into problems. In particular, at half-filling the spectral weight obtained after a resummation of diagrams appears to be negative near frequencies $\omega_{d}= \pm U / 2[13]$. This drawback is connected with divergencies in cumulants at these frequencies [14]. As can be seen from formulas given below, all higher-order cumulants have such divergencies at $\omega_{d}$ with signchanging residues, which are expected to compensate the negative spectral weight in the entire series. On the other hand, at frequencies close to $\omega_{d}$ cumulants are regular. If a selected subset of diagrams is expected to give a correct estimate of the entire series for these frequencies the 
values at $\omega_{d}$ can be corrected by an interpolation using the results for the regular regions. This procedure was applied in reference [14] for the case of two dimensions, half-filling, nearest-neighbor hopping and with the use of the one-loop approximation. The spectrum was shown to consist of four bands. These band structures and the calculated shapes of the electron spectral function are close to those obtained in the Monte-Carlo [16-18], cluster perturbation [19] and the two-particle self-consistent [20] calculations, provided that the temperature is high enough to ensure a short magnetic correlation length. The four-band structure of the spectrum of the Hubbard model was also considered in reference [21].

In the approach of reference [14] the mentioned four-band structure of the spectrum at halffilling has its origin in the regions of large damping which separate the low- and high-frequency bands. In the major part of the Brillouin zone both these types of the bands owe their origin to the same terms of the irreducible part, i.e. to the same interaction processes. This brings up the questions: How this spectrum changes with filling and how the quasiparticle peak, which determines the photoemission leading edge, arises in the spectrum? As expected, this peak differs in nature from other spectral features. In the present work it is shown that at certain deviation from half-filling an additional narrow band arises near the Fermi level on the background of the above-mentioned four bands. The dispersion of the new band, its bandwidth and the variation with filling are close to those of the spin-polaron band in the $t$ - $J$ model $[22,23]$.

In the following section the perturbation expansion for the electron Green's function is formulated in the form convenient for calculations and Larkin's equation is derived. In section 3 the equations of the previous section are used in calculating the spectral function and the obtained results are discussed. Concluding remarks are presented in section 4 .

\section{Diagram technique}

The present work considers the electron Green's function,

$$
G\left(\mathbf{n}^{\prime} \tau^{\prime}, \mathbf{n} \tau\right)=\left\langle\mathcal{T} \bar{a}_{\mathbf{n}^{\prime} \sigma}\left(\tau^{\prime}\right) a_{\mathbf{n} \sigma}(\tau)\right\rangle
$$

Here the angular brackets denote the statistical averaging with the Hamiltonian $\mathcal{H}=H-\mu \sum_{\mathbf{n} \sigma} n_{\mathbf{n} \sigma}$, $\mu$ is the chemical potential, $\mathcal{T}$ is the time-ordering operator which arranges other operators from right to left in ascending order of times $\tau, a_{\mathbf{n} \sigma}(\tau)=\exp (\mathcal{H} \tau) a_{\mathbf{n} \sigma} \exp (-\mathcal{H} \tau)$ and $\bar{a}_{\mathbf{n} \sigma}(\tau)=$ $\exp (\mathcal{H} \tau) a_{\mathbf{n} \sigma}^{\dagger} \exp (-\mathcal{H} \tau)$. Choosing

$$
H_{0}=\frac{U}{2} \sum_{\mathbf{n} \sigma} n_{\mathbf{n} \sigma} n_{\mathbf{n},-\sigma}-\mu \sum_{\mathbf{n} \sigma} n_{\mathbf{n} \sigma} \text { and } H_{1}=\sum_{\mathbf{n} \mathbf{m} \sigma} t_{\mathbf{n} \mathbf{m}} a_{\mathbf{n} \sigma}^{\dagger} a_{\mathbf{m} \sigma}
$$

as the unperturbed Hamiltonian and the perturbation, respectively, and using the known expansion [24] for the evolution operator we get

$$
\begin{aligned}
G\left(\mathbf{n}^{\prime} \tau^{\prime}, \mathbf{n} \tau\right)= & \sum_{k=0}^{\infty} \frac{(-1)^{k}}{k !} \int \ldots \int_{0}^{\beta} \mathrm{d} \tau_{1} \ldots \mathrm{d} \tau_{k} \sum_{\mathbf{n}_{1} \mathbf{n}_{1}^{\prime} \sigma_{1}} \ldots \sum_{\mathbf{n}_{k} \mathbf{n}_{k}^{\prime} \sigma_{k}} t_{\mathbf{n}_{1} \mathbf{n}_{1}^{\prime}} \ldots t_{\mathbf{n}_{k} \mathbf{n}_{k}^{\prime}} \\
& \times\left\langle\mathcal{T} \bar{a}_{\mathbf{n}^{\prime} \sigma}\left(\tau^{\prime}\right) a_{\mathbf{n} \sigma}(\tau) \bar{a}_{\mathbf{n}_{1}^{\prime} \sigma_{1}}\left(\tau_{1}\right) a_{\mathbf{n}_{1} \sigma_{1}}\left(\tau_{1}\right) \ldots \bar{a}_{\mathbf{n}_{k}^{\prime} \sigma_{k}}\left(\tau_{k}\right) a_{\mathbf{n}_{k} \sigma_{k}}\left(\tau_{k}\right)\right\rangle_{0 \mathrm{c}}
\end{aligned}
$$

where $\beta=T^{-1}$ is the inverse temperature, the subscript " 0 " near the angular bracket indicates that the averaging and time dependencies of operators are determined with the Hamiltonian $H_{0}$. The subscript "c" indicates that terms which split into two or more disconnected averages should be dropped out.

In equation (4), the average in the $k$-th order term which contains $k+1$ creation and annihilation operators can be represented by the sum of all possible products of cumulants [25] with the sum of orders equal to $k+1[10,11,14]$. All possible distributions of operators between the cumulants in these products should be taken into account. The sign of a term in this sum is determined by the number of permutations of fermion operators, which bring the sequence of operators in the initial average to that in the term. The order of a cumulant is determined by the numbers of creation or 
annihilation operators in it. These numbers should be equal for the cumulant to be nonzero. Since the Hamiltonian $H_{0}$ in equation (3) is diagonal in the site representation, operators in cumulants belong to the same lattice site. Therefore, due to the translation symmetry of the problem, the cumulant does not depend on the site index. Cumulants of the first and second order read

$$
\begin{aligned}
K_{1}\left(\tau^{\prime} \sigma^{\prime}, \tau \sigma\right)= & \left\langle\mathcal{T} \bar{a}_{\sigma}\left(\tau^{\prime}\right) a_{\sigma}(\tau)\right\rangle_{0} \delta_{\sigma \sigma^{\prime}} \\
K_{2}\left(\tau^{\prime} \sigma, \tau \sigma, \tau_{1}^{\prime} \sigma_{1}, \tau_{1} \sigma_{1}\right)= & \left\langle\mathcal{T} \bar{a}_{\sigma}\left(\tau^{\prime}\right) a_{\sigma}(\tau) \bar{a}_{\sigma_{1}}\left(\tau_{1}^{\prime}\right) a_{\sigma_{1}}\left(\tau_{1}\right)\right\rangle_{0} \\
& -K_{1}\left(\tau^{\prime} \sigma, \tau \sigma\right) K_{1}\left(\tau_{1}^{\prime} \sigma_{1}, \tau_{1} \sigma_{1}\right)+K_{1}\left(\tau^{\prime} \sigma, \tau_{1} \sigma_{1}\right) K_{1}\left(\tau_{1}^{\prime} \sigma_{1}, \tau \sigma\right),
\end{aligned}
$$

where the site indices are dropped.

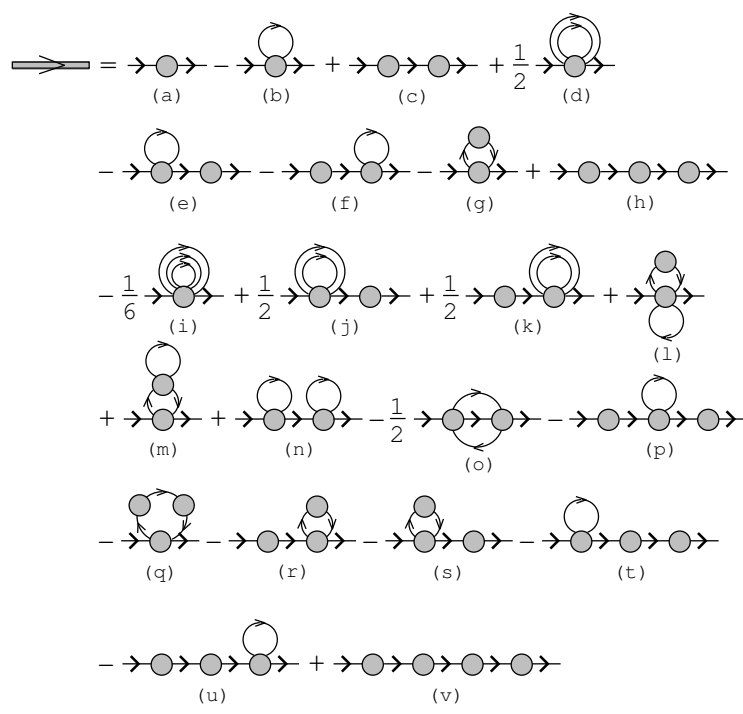

Figure 1. Diagrams of the first four orders of expansion (4).
Actually the above statements determine the rules of the diagram technique. Additionally one has to take into account the presence of topologically equivalent terms i.e., the terms which differ only by permutation of operators $H_{1}\left(\tau_{i}\right)$ in equation (4). Since these terms are equal, in the expansion only one of them can be taken into account with the prefactor $\nu=j / k$ ! where $j$ is the number of topologically equivalent terms. Following reference [11] in diagrams a cumulant is denoted by a circle and the hopping constant $t_{\mathbf{n n}^{\prime}}$ by a line directed from $\mathbf{n}^{\prime}$ to n. The external operators $\bar{a}_{\mathbf{n}^{\prime} \sigma}\left(\tau^{\prime}\right)$ and $a_{\mathbf{n} \sigma}(\tau)$ are denoted by directed lines leaving the cumulant and entering it. The order of a cumulant is equal to a number of incoming or outgoing lines. Summations and integrations over the internal indices $\mathbf{n}_{i}, \mathbf{n}_{i}^{\prime}, \sigma_{i}$ and $\tau_{i}$ are carried out. Since site indices of operators included in a cumulant coincide, some site summations disappear. Also some summations over $\sigma_{i}$ get lost, because in any cumulant, the spin indices of creation and annihilation operators should match. Taking into account the multiplier $(-1)^{k}$ in equation (4), the sign of the diagram is equal to $(-1)^{l}$ where $l$ is the number of loops formed by hopping lines. Figure 1 demonstrates the connected diagrams of the first four orders of the power expansion (4) with their signs and prefactors. Here the thick line with arrow on the left-hand side of the equation is the total Green's function. Notice that if we set $t_{\mathbf{n n}}=0$, contributions of the diagrams (b), (d)-(f), (i)-(n), (p), (t), and (u) vanish. However, below a renormalized hopping parameter will be introduced which is nonzero for the coinciding site indices and therefore the mentioned diagrams are retained in figure 1.

All diagrams can be separated into two categories - those that can be divided into two parts by cutting some hopping line and those that cannot be divided in this way $[8,15]$. These latter diagrams are referred to as irreducible diagrams. Denoting the sum of all irreducible diagrams by $K\left(\mathbf{n}^{\prime} \tau^{\prime}, \mathbf{n} \tau\right)$, after the Fourier transformation we find the following Larkin equation for Green's function:

$$
G\left(\mathbf{k}, \mathrm{i} \omega_{l}\right)=\frac{K\left(\mathbf{k}, \mathrm{i} \omega_{l}\right)}{1-t_{\mathbf{k}} K\left(\mathbf{k}, \mathrm{i} \omega_{l}\right)},
$$

where $t_{\mathbf{k}}=\sum_{\mathbf{n}} \mathrm{e}^{-\mathbf{i} \mathbf{k}(\mathbf{n}-\mathbf{m})} t_{\mathbf{n m}}, \omega_{l}=(2 l+1) \pi T$ is the Matsubara frequency with an integer $l$.

The partial summation can be carried out in the hopping lines of irreducible diagrams by inserting them into the lines. In doing so the hopping constant $t_{\mathbf{k}}$ in the respective formulas is substituted by

$$
\Theta\left(\mathbf{k}, \mathrm{i} \omega_{l}\right)=\frac{t_{\mathbf{k}}}{1-t_{\mathbf{k}} K\left(\mathbf{k}, \mathrm{i} \omega_{l}\right)}=t_{\mathbf{k}}+t_{\mathbf{k}}^{2} G\left(\mathbf{k}, \mathrm{i} \omega_{l}\right)
$$


In the approximation used below the total collection of irreducible diagrams $K\left(\mathbf{k}, \mathrm{i} \omega_{l}\right)$ is substituted by the sum of the two diagrams (a) and (b) in figure 1, which appear in the first two orders of the perturbation theory. Due to the form of the latter diagram this approximation is referred to as the one-loop approximation. In the diagram (b) the hopping line is renormalized in accordance with equation (7). Thus,

$$
K\left(\mathrm{i} \omega_{l}\right)=K_{1}\left(\mathrm{i} \omega_{l}\right)-T \sum_{l_{1} \sigma_{1}} K_{2}\left(\mathrm{i} \omega_{l} \sigma, \mathrm{i} \omega_{l_{1}} \sigma_{1}, \mathrm{i} \omega_{l_{1}} \sigma_{1}\right) \frac{1}{N} \sum_{\mathbf{k}} t_{\mathbf{k}}^{2} G\left(\mathbf{k}, \mathrm{i} \omega_{l_{1}}\right),
$$

where $K_{1}\left(\mathrm{i} \omega_{l}\right)$ and

$$
\begin{aligned}
& K_{2}\left(\mathrm{i} \omega_{l^{\prime}} \sigma, \mathrm{i} \omega_{l} \sigma, \mathrm{i} \omega_{l_{1}^{\prime}} \sigma_{1}, \mathrm{i} \omega_{l_{1}} \sigma_{1}\right)= \\
& \quad=\iiint \int_{0}^{\beta} \mathrm{d} \tau^{\prime} \mathrm{d} \tau \mathrm{d} \tau_{1}^{\prime} \mathrm{d} \tau_{1} \mathrm{e}^{-\mathrm{i} \omega_{l^{\prime}} \tau^{\prime}+\mathrm{i} \omega_{l} \tau-\mathrm{i} \omega_{l_{1}^{\prime}} \tau_{1}^{\prime}+\mathrm{i} \omega_{l_{1}} \tau_{1}} K_{2}\left(\tau^{\prime} \sigma, \tau \sigma, \tau_{1}^{\prime} \sigma_{1}, \tau_{1} \sigma_{1}\right) \\
& =\beta \delta_{l+l_{1}, l^{\prime}+l_{1}^{\prime}} K_{2}\left(\mathrm{i} \omega_{l} \sigma, \mathrm{i} \omega_{l_{1}^{\prime}} \sigma_{1}, \mathrm{i} \omega_{l_{1}} \sigma_{1}\right)
\end{aligned}
$$

are the Fourier transforms of cumulants $(5), N$ is the number of sites and we set $\sum_{\mathbf{k}} t_{\mathbf{k}}=0$. Notice that in this approximation $K$ does not depend on momentum.

Now we need to calculate the cumulants in equation (8). To do this it is convenient to introduce the Hubbard operators $X_{\mathbf{n}}^{i j}=|i \mathbf{n}\rangle\langle j \mathbf{n}|$ where $|i \mathbf{n}\rangle$ are eigenvectors of site Hamiltonians forming $H_{0}$, equation (3). For each site there are four states: the empty state $|0 \mathbf{n}\rangle$ with the energy $E_{0}=0$, the two degenerate singly occupied states $|\sigma \mathbf{n}\rangle$ with the energy $E_{1}=-\mu$ and the doubly occupied state $|2 \mathbf{n}\rangle$ with the energy $E_{2}=U-2 \mu$. The Hubbard operators are connected by the relations

$$
a_{\mathbf{n} \sigma}=X_{\mathbf{n}}^{0 \sigma}+\sigma X_{\mathbf{n}}^{-\sigma, 2}, \quad a_{\mathbf{n} \sigma}^{\dagger}=X_{\mathbf{n}}^{\sigma 0}+\sigma X_{\mathbf{n}}^{2,-\sigma}
$$

with the creation and annihilation operators. The commutation relations for the Hubbard operators are easily derived from their definition. Using equation (9) the first cumulant in equation (5) can be computed straightforwardly:

$$
K_{1}\left(\mathrm{i} \omega_{l}\right)=\frac{1}{Z_{0}}\left(\frac{\mathrm{e}^{-\beta E_{\sigma}}+\mathrm{e}^{-\beta E_{0}}}{\mathrm{i} \omega_{l}-E_{\sigma 0}}+\frac{\mathrm{e}^{-\beta E_{2}}+\mathrm{e}^{-\beta E_{\sigma}}}{\mathrm{i} \omega_{l}-E_{2 \sigma}}\right),
$$

where $Z_{0}=\mathrm{e}^{-\beta E_{0}}+2 \mathrm{e}^{-\beta E_{\sigma}}+\mathrm{e}^{-\beta E_{2}}$ is the site partition function and $E_{i j}=E_{i}-E_{j}$. As indicated in references $[8,10,13]$, if $K\left(\mathbf{k}, \mathrm{i} \omega_{l}\right)$ is approximated by this cumulant the resulting Green's function (6) corresponds to the Hubbard-I approximation [2].

To calculate $K_{2}$ it is convenient to use Wick's theorem for Hubbard operators [5-8]:

$$
\begin{aligned}
& \left\langle\mathcal{T} X_{\alpha_{1}}\left(\tau_{1}\right) \ldots X_{\alpha_{i}}\left(\tau_{i}\right) X_{\alpha}(\tau) X_{\alpha_{i+1}}\left(\tau_{i+1}\right) \ldots X_{\alpha_{n}}\left(\tau_{n}\right)\right\rangle_{0}= \\
& \quad=\sum_{k=1}^{n}(-1)^{P_{k}} g_{\alpha}\left(\tau-\tau_{k}\right)\left\langle\mathcal{T} X_{\alpha_{1}}\left(\tau_{1}\right) \ldots\left[X_{\alpha_{k}}, X_{\alpha}\right]_{ \pm}\left(\tau_{k}\right) \ldots X_{\alpha_{n}}\left(\tau_{n}\right)\right\rangle_{0}
\end{aligned}
$$

where $\alpha$ is the index combining the state and site indices of the Hubbard operator. If $X_{\alpha}$ is a fermion operator ( $X^{0 \sigma}, X^{\sigma 2}$ and their conjugates), $P_{k}$ is the number of permutation with other fermion operators which is necessary to transfer the operator $X_{\alpha}$ from its position on the left-hand side of equation (11) to the position on the right-hand side. In this case

$$
g_{\alpha}(\tau)=\frac{\mathrm{e}^{E_{i j} \tau}}{\mathrm{e}^{\beta E_{i j}}+1}\left\{\begin{aligned}
-1, & \tau>0 \\
\mathrm{e}^{\beta E_{i j}}, & \tau<0
\end{aligned}\right.
$$

where $i$ and $j$ are the state indices of $X_{\alpha}$. If $X_{\alpha}$ is a boson operator $\left(X^{00}, X^{22}, X^{\sigma \sigma^{\prime}}, X^{02}\right.$, and $\left.X^{20}\right), P_{k}=0$ and

$$
g_{\alpha}(\tau)=\frac{\mathrm{e}^{E_{i j} \tau}}{\mathrm{e}^{\beta E_{i j}}-1}\left\{\begin{aligned}
1, & \tau>0 \\
\mathrm{e}^{\beta E_{i j}}, & \tau<0
\end{aligned}\right.
$$


In equation (11), $\left[X_{\alpha_{k}}, X_{\alpha}\right]_{ \pm}$denotes an anticommutator when both operators are of fermion type and a commutator in other cases.

The substitution of equation (9) in $\left\langle\mathcal{T} \bar{a}_{\sigma}\left(\tau^{\prime}\right) a_{\sigma}(\tau) \bar{a}_{\sigma_{1}}\left(\tau_{1}^{\prime}\right) a_{\sigma_{1}}\left(\tau_{1}\right)\right\rangle$ in $K_{2}$, equation (5), leads to six nonvanishing averages of Hubbard operators (such averages are nonzero if the numbers of the operators $X^{0 \sigma}$ and $X^{\sigma 0}$ coincide therein, and the same is true for the pair $X^{\sigma 2}$ and $X^{2 \sigma}$ ). Applying Wick's theorem (11) to these averages the number of operators therein is sequentially decreased until only time-independent operators are left. For $H_{0}$ in equation (3) these are $X^{00}, X^{\sigma \sigma^{\prime}}$, and $X^{22}$. Their averages are easily calculated. As a result, after some algebra we find

$$
\begin{aligned}
\sum_{\sigma_{1}} & K_{2}\left(\mathrm{i} \omega_{l} \sigma, \mathrm{i} \omega_{l_{1}} \sigma_{1}, \mathrm{i} \omega_{l_{1}} \sigma_{1}\right)=-Z_{0}^{-1} U\left\{\mathrm{e}^{-\beta E_{0}} g_{0 \sigma}\left(\mathrm{i} \omega_{l}\right) g_{0 \sigma}\left(\mathrm{i} \omega_{l_{1}}\right) g_{02}\left(\mathrm{i} \omega_{l}+\mathrm{i} \omega_{l_{1}}\right)\left[g_{0 \sigma}\left(\mathrm{i} \omega_{l}\right)+g_{0 \sigma}\left(\mathrm{i} \omega_{l_{1}}\right)\right]\right. \\
& +\mathrm{e}^{-\beta E_{2}} g_{\sigma 2}\left(\mathrm{i} \omega_{l}\right) g_{\sigma 2}\left(\mathrm{i} \omega_{l_{1}}\right) g_{02}\left(\mathrm{i} \omega_{l}+\mathrm{i} \omega_{l_{1}}\right)\left[g_{\sigma 2}\left(\mathrm{i} \omega_{l}\right)+g_{\sigma 2}\left(\mathrm{i} \omega_{l_{1}}\right)\right] \\
& \left.+\mathrm{e}^{-\beta E_{1}}\left[g_{0 \sigma}\left(\mathrm{i} \omega_{l}\right) g_{\sigma 2}\left(\mathrm{i} \omega_{l}\right)\left(g_{0 \sigma}\left(\mathrm{i} \omega_{l_{1}}\right)-g_{\sigma 2}\left(\mathrm{i} \omega_{l_{1}}\right)\right)^{2}+g_{0 \sigma}\left(\mathrm{i} \omega_{l_{1}}\right) g_{\sigma 2}\left(\mathrm{i} \omega_{l_{1}}\right)\left(g_{0 \sigma}^{2}\left(\mathrm{i} \omega_{l}\right)+g_{\sigma 2}^{2}\left(\mathrm{i} \omega_{l}\right)\right)\right]\right\} \\
& -Z_{0}^{-2} U^{2} \beta \delta_{l l_{1}}\left(\mathrm{e}^{-\beta\left(E_{0}+E_{2}\right)}+2 \mathrm{e}^{-\beta\left(E_{0}+E_{1}\right)}+3 \mathrm{e}^{-2 \beta E_{1}}+2 \mathrm{e}^{-\beta\left(E_{1}+E_{2}\right)}\right) g_{0 \sigma}^{2}\left(\mathrm{i} \omega_{l}\right) g_{\sigma 2}^{2}\left(i \omega_{l}\right) \\
& +Z_{0}^{-2} U^{2} \beta\left(2 \mathrm{e}^{-\beta\left(E_{0}+E_{2}\right)}+\mathrm{e}^{-\beta\left(E_{0}+E_{1}\right)}+\mathrm{e}^{-\beta\left(E_{1}+E_{2}\right)}\right) g_{0 \sigma}\left(\mathrm{i} \omega_{l}\right) g_{\sigma 2}\left(\mathrm{i} \omega_{l}\right) g_{0 \sigma}\left(\mathrm{i} \omega_{l_{1}}\right) g_{\sigma 2}\left(\mathrm{i} \omega_{l_{1}}\right), \quad
\end{aligned}
$$

where $g_{i j}\left(\mathrm{i} \omega_{l}\right)=\left(\mathrm{i} \omega_{l}+E_{i j}\right)^{-1}$ is the Fourier transform of functions (12) and (13).

Equations (10) and (14) can be significantly simplified for the case of principal interest $U \gg T$. In this case if $\mu$ satisfies the conditions

$$
\lambda<\mu<U-\lambda,
$$

$\lambda \gg T$, the exponent $\exp \left(-\beta E_{1}\right)$ is much larger than $\exp \left(-\beta E_{0}\right)$ and $\exp \left(-\beta E_{2}\right)$. By passing to real frequencies we can ascertain that terms in (14) with the two latter multipliers have the same peculiarities as other terms. Therefore the terms with these multipliers can be omitted in the equations and we get

$$
\begin{aligned}
& K_{1}\left(\mathrm{i} \omega_{l}\right)=\frac{\mathrm{i} \omega_{l}+\mu-U / 2}{\left(\mathrm{i} \omega_{l}+\mu\right)\left(\mathrm{i} \omega_{l}+\mu-U\right)}, \\
& \sum_{\sigma_{1}} K_{2}\left(\mathrm{i} \omega_{l} \sigma, \mathrm{i} \omega_{l_{1}} \sigma_{1}, \mathrm{i} \omega_{l_{1}} \sigma_{1}\right)=-\frac{1}{2} U g_{0 \sigma}\left(\mathrm{i} \omega_{l}\right) g_{\sigma 2}\left(\mathrm{i} \omega_{l}\right)\left[g_{0 \sigma}^{2}\left(\mathrm{i} \omega_{l_{1}}\right)+g_{\sigma 2}^{2}\left(\mathrm{i} \omega_{l_{1}}\right)\right] \\
& \quad-\frac{1}{2} U g_{0 \sigma}\left(\mathrm{i} \omega_{l_{1}}\right) g_{\sigma 2}\left(\mathrm{i} \omega_{l_{1}}\right)\left[g_{0 \sigma}\left(\mathrm{i} \omega_{l}\right)-g_{\sigma 2}\left(\mathrm{i} \omega_{l}\right)\right]^{2}-\frac{3}{4} U^{2} \beta \delta_{l l_{1}} g_{0 \sigma}^{2}\left(\mathrm{i} \omega_{l}\right) g_{\sigma 2}^{2}\left(\mathrm{i} \omega_{l}\right) .
\end{aligned}
$$

Let us turn to real frequencies by substituting $\mathrm{i} \omega_{l}$ with $z=\omega+\mathrm{i} \eta$ where $\eta$ is a small positive constant which affords an artificial broadening possible. Results given in the next section were calculated with $G(\mathbf{k}, z)$ on the right-hand side of equation (8) taken from the Hubbard-I approximation. As mentioned, this Green's function is obtained if $K(\mathbf{k} \omega)$ in equation (6) is approximated by $K_{1}$ from equation (16) which gives

$$
\begin{aligned}
G(\mathbf{k}, z) & =\frac{1}{2}\left(1+\frac{t_{\mathbf{k}}}{\sqrt{U^{2}+t_{\mathbf{k}}^{2}}}\right) \frac{1}{z-\varepsilon_{1, \mathbf{k}}}+\frac{1}{2}\left(1-\frac{t_{\mathbf{k}}}{\sqrt{U^{2}+t_{\mathbf{k}}^{2}}}\right) \frac{1}{z-\varepsilon_{2, \mathbf{k}}} \\
\varepsilon_{1, \mathbf{k}} & =\frac{1}{2}\left(U+t_{\mathbf{k}}+\sqrt{U^{2}+t_{\mathbf{k}}^{2}}\right)-\mu, \quad \varepsilon_{2, \mathbf{k}}=\frac{1}{2}\left(U+t_{\mathbf{k}}-\sqrt{U^{2}+t_{\mathbf{k}}^{2}}\right)-\mu .
\end{aligned}
$$

Below the two-dimensional square lattice is considered. It is supposed that only the hopping constants between the nearest neighbor sites $t$ are nonzero which gives $t_{\mathbf{k}}=2 t\left[\cos \left(k_{x}\right)+\cos \left(k_{y}\right)\right]$ where the intersite distance is taken as the unit of length. Due to the electron-hole symmetry in this case the consideration can be restricted to the range of the chemical potentials $\mu \leqslant U / 2$. 


\section{Spectral function}

Figure 2 demonstrates $\Im K(\omega)$ calculated with the use of equations (8), (16) and (17). The change to real frequencies carried out in the previous section converts the Matsubara function (2) into the retarded Green's function [24]. It is an analytic function in the upper half-plane which requires that $\Im K(\omega)$ be negative. As seen in figure 2, this condition is violated at $\omega_{d}=-\mu$ and
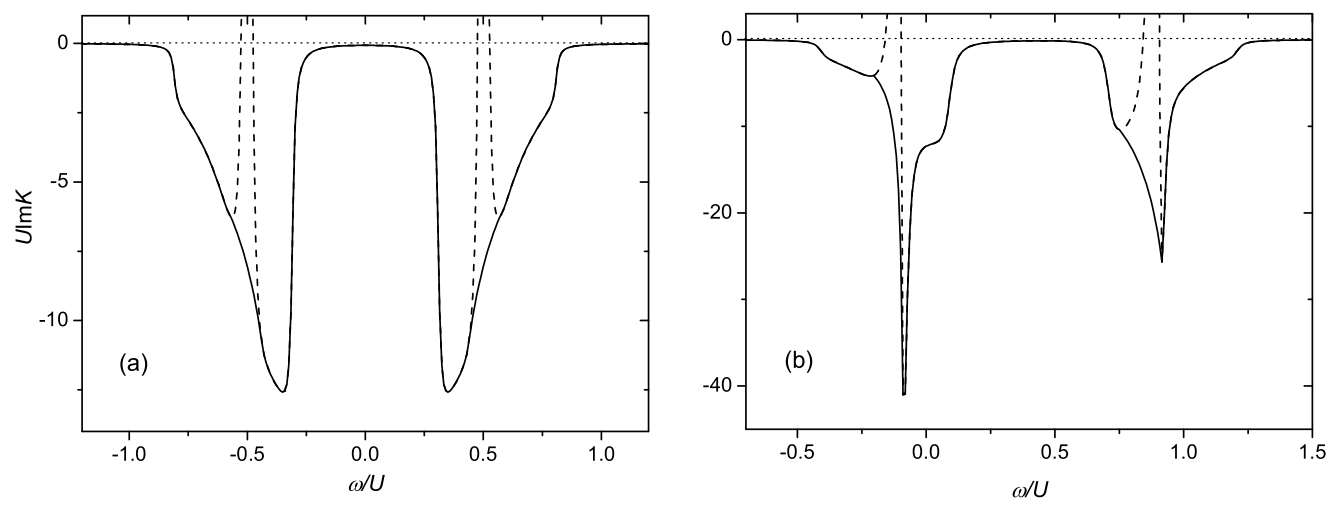

Figure 2. The imaginary part of $K(\omega)$ calculated using equations (8), (16) and (17) for a $100 \times 100$ lattice, $t=-U / 8$ and $T=0.001 U$ (the dashed lines). (a) $\mu=0.5 U, \eta=0.01 U$. (b) $\mu=0.1 U$, $\eta=0.02 U$. The solid lines show the corrected $\Im K(\omega)$ (see text).

$U-\mu$. This difficulty of the considered approximation was indicated in reference [13]. The problem is connected with divergencies at these frequencies introduced by functions $g_{0 \sigma}(\omega)$ and $g_{\sigma 2}(\omega)$ in the above formulas. As can be seen from the procedure of calculating the cumulants in the previous section, these functions and divergencies with sign-changing residues appear in all orders of the perturbation expansion (4). In the entire series the divergencies are expected to compensate each other so that the resulting $\Im K(\omega)$ is negative everywhere. However, in the considered subset of terms such compensation does not occur. Nevertheless, as seen in figure 2, at frequencies close to $\omega_{d} \Im K(\omega)$ is negative. If the used subset of diagrams is expected to give a correct estimate of the entire series for these frequencies, the values of $\Im K(\omega)$ for the problem frequency domains where $\Im K(\omega)>0$ can be reconstructed using an interpolation and the values of $\Im K(\omega)$ in the regular region. For this purpose different interpolating functions were tested. Since the regions where $\Im K(\omega)>0$ are connected with poles, their widths can be decreased by decreasing the artificial broadening $\eta$. This provides a way of testing the suitability of interpolation functions and the choice of supporting points used in determining the free parameters in the functions. The points were chosen away from the regions where $\Im K(\omega)>0$. The functions and supporting points were adopted in such a way that the sharp minima in $\Im K(\omega)$ were not blurred. It was found that in this case the choice of the interpolating function and supporting points only weakly effects the shape of the spectral function discussed below. Examples of the interpolations used are given in figure 2 .

As seen in figure 2a, at half-filling, $\mu=U / 2, \Im K(\omega)$ has two broad minima. With the chemical potential decreasing from this value, these minima shift with respect to the Fermi level without a noticeable change of their shapes until the Fermi level enters one of the minima at $\mu \approx 0.17 U$. As this takes place, two new sharp minima arise near the frequencies $-\mu$ and $U-\mu$ on the background of the above-mentioned broad minima. The appearance of the broad features in figure 2 is connected with the third term in $\sum_{\sigma_{1}} K_{2}$ in equation (16), while the sharp minima are related to the second term in this formula. Its contribution to $K(\omega)$, equation (8), grows rapidly when the Fermi level enters the broad minimum.

The function $K(z)$ should be analytic in the upper half-plane as well and therefore its real part can be calculated from its imaginary part using the Kramers-Kronig relations. We use this way with the interpolated $\Im K(\omega)$ to avoid the effect of the divergencies on $\Re K(\omega)$. However, the use of the interpolation somewhat overrates the values of $|\Im K(\omega)|$ which leads to the overestimation 
of the tails in the real part. To correct this defect the interpolated $K(\omega)$ is scaled so that in the far tails its real part coincides with the values obtained from equation (8).

The spectral function

$$
A(\mathbf{k} \omega)=-\frac{1}{\pi} \Im G(\mathbf{k} \omega)=-\frac{1}{\pi} \frac{\Im K(\omega)}{\left[1-t_{\mathbf{k}} \Re K(\omega)\right]^{2}+\left[t_{\mathbf{k}} \Im K(\omega)\right]^{2}}
$$

obtained in this way for the momenta along the symmetry lines of the square Brillouin zone is shown in figure 3 . The shapes of the spectral function in figure $3 \mathrm{a}$ are nearly the same as at half-
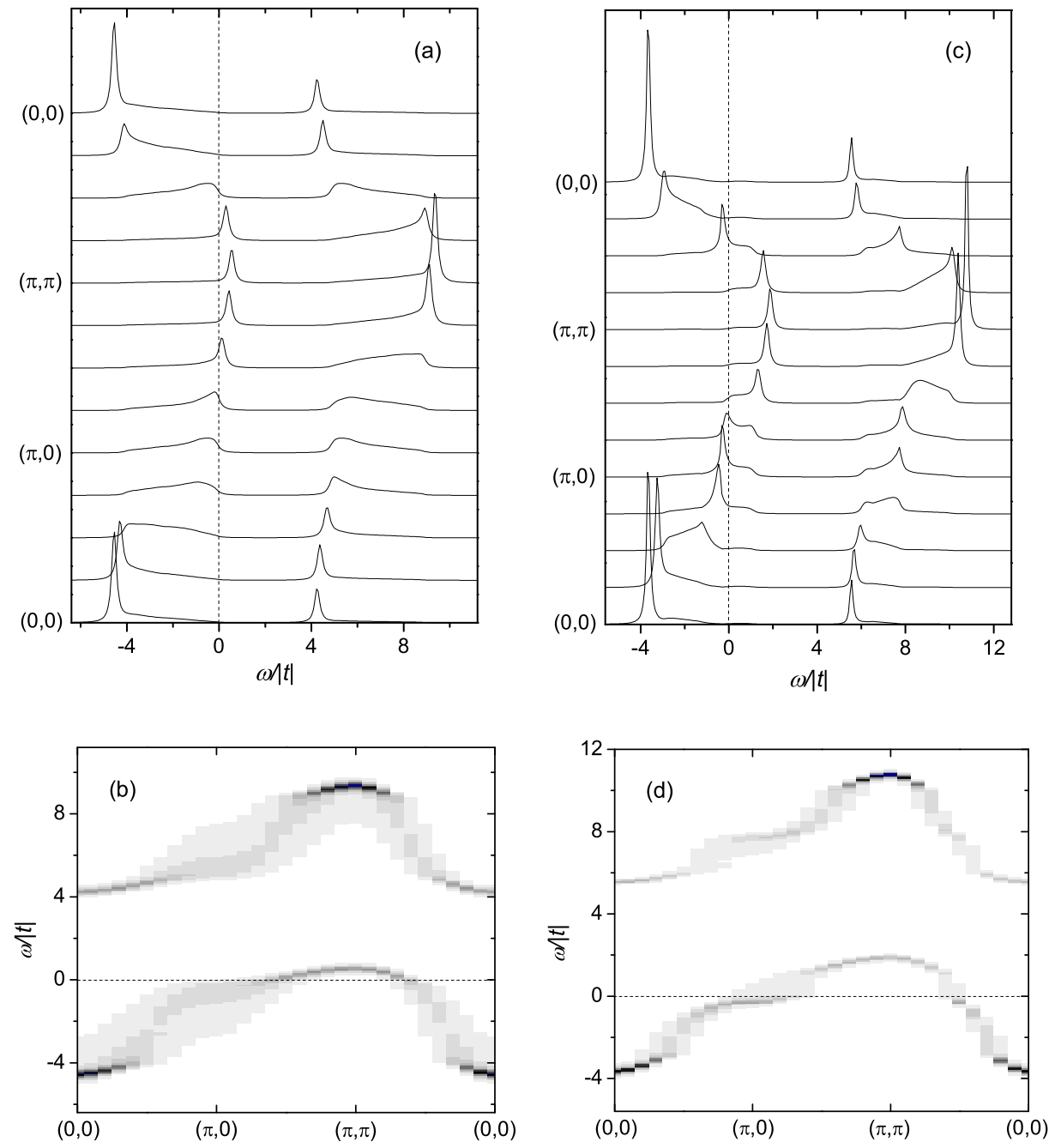

Figure 3. Panels (a) and (c): the spectral function $A(\mathbf{k} \omega)$ calculated for momenta along the symmetry lines of the square Brillouin zone in a $100 \times 100$ lattice for $t=-U / 8, T=0.001 U$, $\eta=0.02 U, \mu=0.2 U$ (a) and $\mu=0.05 U$ (c). The dispersions of maxima in the panels (a) and (c) are shown in the panels (b) and (d), respectively. Here darker areas correspond to larger intensities of maxima.

filling - with decreasing $\mu$ from $0.5 U$ to approximately $0.17 U$ these curves shift with respect to the Fermi level without perceptible changes in their shapes. As indicated in reference [14], four bands can be distinguished in these spectra. For parameters of figure 3a these bands are located near frequencies $-4|t|,|t|, 4|t|$, and $9|t|$ (see figure $3 \mathrm{~b}$ ). For the major part of the Brillouin zone the peaks forming the bands arise at frequencies which satisfy the equation $1-t_{\mathbf{k}} \Re K(\omega)=0$ and fall into the region of a small damping $|\Im K(\omega)|$ [see equation (18)]. As seen in figure 2, such regions of 
small damping are located between and on the outside of the two broad minima in $\Im K(\omega)$. This is the reason of the existence of the four well separated bands - two of them are located between the minima of $\Im K(\omega)$, while two others are on the outside of these minima. Broader maxima of $A(\mathbf{k} \omega)$ for the momenta near the boundary of the magnetic Brillouin zone are of different nature - since $t_{\mathbf{k}}$ is small for such momenta, the resonant denominator in equation (18) does not vanish and the shape of the spectral functions is determined by $\varsigma K(\omega)$ in the numerator of this formula.

More substantial changes in $A(\mathbf{k} \omega)$ occur for $\mu \leqslant 0.17 U$ when the Fermi level enters one of the broad minima in $\Im K(\omega)$. As seen from figure 3c, in addition to the mentioned four bands there appear sharp dispersive features near $\omega=-\mu$ and $U-\mu$ for the momenta in the vicinity of the boundary of the magnetic Brillouin zone. It is clear that these changes in the spectral function are connected with the changes in $\Im K(\omega)$ shown in figure 2 . The peaks near $-\mu$ are more intensive than those near $U-\mu$ and are located in the nearest vicinity of the Fermi level. For $\mu \approx 0.17 U$ the width of the band formed by the former peaks is comparable to the superexchange constant $J=4 t^{2} / U$ of the effective Heisenberg model which describes magnetic excitations in the limit $U \gg|t|$. This obviously indicates the participation of the spin excitations in the formation of these band states. The bandwidth decreases with further reduction of the electron concentration. As this takes place, the peak intensities first grow and then saturate. The maximum energies of the band are located near the boundary of the magnetic Brillouin zone. For parameters in figure 3c for these momenta, the band touches the Fermi level and the corresponding peaks disappear above it (see figure 3d).

These properties of the band resembles those of the spin-polaron band in the $t$ - $J$ model. This latter band is also located near the Fermi level, has the similar dispersion and the bandwidth, which decreases with the reduction of the electron concentration (with the increase of the hole doping counted from half-filling) [23]. However, there is one essential difference in the behavior of these bands in the Hubbard and $t-J$ models. In the former model the narrow band near the Fermi level appears at a certain deficiency of electrons, while the above-mentioned four bands exist in the entire range of electron concentrations considered. In the $t$ - $J$ model the situation is opposite - the spin-polaron band exists in the wide range of hole concentrations $0 \leqslant x \leqslant 0.17$, while the wider band - an analog of the four-band structure - starts to form at $x \approx 0.06$ [23].

The value of the chemical potential for which the Fermi level enters the minimum of $\Im K(\omega)$ and the narrow band begins to form, depends on the ratio $t / U$. For example, for $t=-U / 4$ this happens at $\mu \approx 0.27 U$.

There is some difficulty in comparing our results with the data of Monte-Carlo calculations and cluster theories. This is due to the fact mentioned in reference [14]: the one-loop approximation overestimates the spectral weights of the two internal bands of the four-band structure near the momenta $(0,0)$ and $(\pi, \pi)$. As a result, the electron concentration calculated from the formula

$$
\langle n\rangle=\frac{2}{N} \sum_{\mathbf{k}} \int_{-\infty}^{\infty} \mathrm{d} \omega \frac{A(\mathbf{k} \omega)}{\exp (\beta \omega)+1}
$$

appears to be considerably underestimated. For example, for the parameters in figures $3 \mathrm{a}$ and $3 \mathrm{c}$ such estimated $\langle n\rangle$ is equal to 0.87 and 0.68 , respectively. From the comparison of the dispersions in figures $3 \mathrm{~b}$ and $3 \mathrm{~d}$ with the results of Monte-Carlo calculations (figure 9 in [18]) it can be concluded that these concentrations should be approximately 0.95 and 0.85 , respectively. With this in mind we find that the spectral functions and dispersions in figure 3 are close to the Monte-Carlo spectra (cf. with figures 10 and 11 in [18]). In these latter spectra, for some deviation from half-filling, a weakly dispersive feature is also observed near the Fermi level. However, this feature has low intensity and is lost at the foot of a more intensive maximum on approaching the boundary of the magnetic Brillouin zone. These differences may be connected with the comparatively high temperature $T=0.33|t|$ used in the Monte-Carlo simulations. A similar weakly dispersive feature near the Fermi level was also obtained in quantum cluster theories, though for much smaller deviations from half-filling (cf. with figure 2c in [27] and figure 32 in [28]). 


\section{Conclusion}

The considered diagram technique is very promising in being generalized to many-band Hubbard models for which energy parameters of the one-site parts of the Hamiltonians exceed or at least are comparable to the intersite parameters. The expansion in powers of these latter parameters can be expressed in terms of cumulants in the same manner as discussed above. Now there are distinct cumulants which belong to different site states characterized by dissimilar parameters of the repulsion and the level energy. These cumulants are described by formulas similar to equations (10) and (14). For example, for the Emery model [29] which describes oxygen $2 p_{\sigma}$ and copper $3 d_{x^{2}-y^{2}}$ orbitals of $\mathrm{Cu}-\mathrm{O}$ planes in high- $T_{\mathrm{c}}$ superconductors there are two types of cumulants corresponding to these states. Diagrams of the lowest orders, e.g., for Green's function on copper sites resemble those shown in figure 1 where "oxygen" cumulants are included in hopping lines. Equations of the type of (6) and partial summations similar to equation (7) can be also derived in this case.

In summary, the diagram technique for the one-band Hubbard model was formulated for the case of moderate to strong Hubbard repulsion. The expansion in powers of the hopping constant is expressed in terms of site cumulants of electron creation and annihilation operators. For Green's function the equation of the Larkin type was derived and solved for the case of two dimensions and nearest-neighbor hopping. With the electron concentration decreasing, in addition to the four bands observed at half-filling, a narrow band arises near the Fermi level for the momenta near the boundary of the magnetic Brillouin zone. On the occurrence, the width of the band is comparable to the superexchange constant $J=4 t^{2} / U$ which indicates the participation of the spin excitations in the band formation. The bandwidth decreases with the electron concentration decreasing. The maximum energies of the band are located near the boundary of the magnetic Brillouin zone. For some deviation from half-filling in these points, the band touches the Fermi level. With these properties the band resembles the spin-polaron band of the $t-J$ model.

\section{References}

1. Gutzwiller M.C., Phys. Rev. Lett., 1963, 10, 159.

2. Hubbard J., Proc. R. Soc. London, Ser. A, 1963, 276, 238.

3. Kanamori J., Prog. Theor. Phys., 1963, 30, 275.

4. Hubbard J., Proc. R. Soc. London, Ser. A, 1966, 296, 82.

5. Westwanski B., Pawlikovski A., Phys. Lett. A, 1973, 43, 201.

6. Slobodyan P.M., Stasyuk I.V., Teor. Mat. Fiz., 1974, 19, 423 (in Russian) [Theor. Mat. Phys., 1974, 19, 616].

7. Zaitsev R.O., ZhETF, 1976, 70, 1100 (in Russian) [Sov. Phys. JETP, 1976, 43, 574].

8. Izyumov Yu.A., Skryabin Yu.N. Statistical Mechanics of Magnetically Ordered Systems. Consultants Bureau, New York, 1988.

9. Ovchinnikov S.G., Valkov V.V. Hubbard operators in the theory of strongly correlated electrons. Imperial College Press, London, 2004.

10. Vladimir M.I., Moskalenko V.A., Teor. Mat. Fiz., 1990, 82, 428 (in Russian) [Theor. Mat. Phys., 1990, 82, 301]; Vakaru S.I., Vladimir M.I., Moskalenko V.A., Teor. Mat. Fiz., 1990, 85, 248 (in Russian) [Theor. Mat. Phys., 1990, 85, 1185].

11. Metzner W., Phys. Rev. B, 1991, 43, 8549.

12. Moskalenko V.A., Entel P., Digor D.F., Phys. Rev. B, 1999, 59, 619.

13. Pairault S., Sénéchal D., Tremblay A.-M.S., Eur. Phys. J. B, 2000, 16, 85.

14. Sherman A., Phys. Rev. B, 2006, 73, 155105.

15. Larkin A.I., ZhETF, 1959, 37, 264 (in Russian) [Sov. Phys. JETP, 1960, 37, 186].

16. Moreo A., Haas S., Sandvik A.W., Dagotto E., Phys. Rev. B, 1995, 51, 12045.

17. Preuss R., Hanke W., von der Linden W., Phys. Rev. Lett., 1995, 75, 1344.

18. Gröber C., Eder R., Hanke W., Phys. Rev. B, 2000, 62, 4336.

19. Dahnken C. et al., Phys. Rev. B, 2004, 70, 245110.

20. Tremblay A.-M.S., Kyung B., Sénéchal D., Fizika Nizkikh Temperatur 2006, 32, 561.

21. Shvaika A.M., Phys. Rev. B, 2000, 62, 2358; Stasyuk I.V., Shvaika A.M., Ukrainian J. Phys., 2002, 47, 975.

22. Plakida N.M, Oudovenko V.S., Yushankhai V.Yu, Phys. Rev. B, 1994, 50, 6431. 
23. Sherman A., Schreiber M., Phys. Rev. B, 1994, 50, 12887; Pseudogaps and magnetic properties of the two-dimensional $t$ - $J$ model. - In: Studies of High Temperature Superconductors, 27, 163. Nova Science Publishers, New York, 1999, ed. Narlikar A.V.; Eur. Phys. J. B, 2003, 32, 203.

24. Abrikosov A.A., Gor'kov L.P., Dzyaloshinskii I.E. Methods of Quantum Field Theory in Statistical Physics. Pergamon Press, New York, 1965.

25. Kubo R., J. Phys. Soc. Jpn., 1962, 17, 1100.

26. Moukouri S. et al., Phys. Rev. B, 2000, 61, 7887.

27. Kyung B. et al., Phys. Rev. B, 2006, 73, 165114

28. Maier T. et al., Rev. Modern Phys., 2005, 77, 1027.

29. Emery V.J., Phys. Rev. Lett., 1987, 58, 2794.

\title{
Еволюція спектру моделі Хаббарда при зміні концентрації
}

\section{О.Шерман}

Інститут фізики, Університет Тарту, Ріа 142, 51014 Тарту, Естонія

Отримано 27 лютого 2006 р., в остаточному вигляді - 12 травня 2006 р.

\begin{abstract}
Сформульовано діаграмну техніку для однозонної моделі Хаббарда для випадку проміжного та сильного хаббардівського відштовхування. Розклад за степенями константи перескоку виражено через вузлові кумулянти електронних операторів народження та знищення. Отримано рівняння Ларкіна для функції Гріна та розв'язано його у однопетлевому наближенні для двовимірного випадку та переносу між найближчими сусідами. При пониженні електронної концентрації крім чотирьох зон, що існують при половинному заповненні, з'являється вузька зона поблизу рівня Фермі. Дисперсія цієї нової зони, її ширина та зміна при зміні концентрації є подібними до таких же величин для випадку спін-поляронної зони в $t-J$ моделі.
\end{abstract}

Ключові слова: модель Хаббарда, діаграмна техніка, енергетичний спектр

PACS: $71.10 . F d, 71.10 .-w$ 